Boise State University

ScholarWorks

$7-1-2010$

Ground-Penetrating-Radar Reflection Attenuation Tomography with an Adaptive Mesh

Emily A. Hinz

Boise State University

John H. Bradford

Boise State University 


\title{
Ground-penetrating-radar reflection attenuation tomography with an adaptive mesh
}

\author{
Emily A. Hinz ${ }^{1}$ and John H. Bradford ${ }^{1}$
}

\begin{abstract}
Ground-penetrating radar (GPR) attenuation-difference analysis can be a useful tool for studying fluid transport in the subsurface. Surface-based reflection attenuation-difference tomography poses a number of challenges that are not faced by crosshole attenuation surveys. We create and analyze a synthetic attenuation-difference GPR data set to determine methods for processing amplitude changes and inverting for conductivity differences from reflection data sets. Instead of using a traditional grid-based inversion, we use a data-driven adaptive-meshing algorithm to alter the model space and to create a more even distribution of resolution. Adaptive meshing provides a method for improving the resolution of the model space while honoring the data limitations and improving the quality of the attenuation difference inversion. Comparing inversions on a conventional rectangular grid with the adaptive mesh, we find that the adaptively meshed model reduces the inversion computation time by an average of $75 \%$ with an improvement in the root mean square error of up to $15 \%$. While the sign of the conductivity change is correctly reproduced by the inversion algorithm, the magnitude varies by as much as much as $50 \%$ from the true values. Our heterogeneous conductivity model indicates that the attenuation difference inversion algorithm effectively locates conductivity changes, and that surface-based reflection surveys can produce models as accurate as traditional crosshole surveys.
\end{abstract}

\section{INTRODUCTION}

Geophysical techniques have gained increasing use for characterizing and monitoring subsurface hydrogeologic transport pathways and mechanisms. Electrical methods such as DC resistivity and lowfrequency electromagnetic (EM) surveying are often used for mapping conductive plumes, and measurements can identify changes in the location and concentration of conductive anomalies (Acworth and Dasey, 2003; Chambers et al., 2003; Kim et al., 2004).

Because intrinsic ground-penetrating-radar (GPR) attenuation depends strongly on electrical conductivity, GPR is also a useful tool for mapping conductive regions in the subsurface. Additionally, some of the properties of GPR, such as its higher frequencies and the wave propagation of the electromagnetic signal, allow for higher lateral and vertical resolution of features than lower-frequency EM techniques. A number of previous studies have investigated the use of GPR in crosshole experiments for detecting and monitoring conductive tracers through attenuation analysis (Liu et al., 1998; DayLewis et al., 2003; Chang et al., 2004; Johnson et al., 2007). These studies demonstrate the practicality and usefulness of GPR attenuation inversion for conductivity estimation, but they also note that the technique is prone to more errors in conductivity estimation than lower-frequency EM techniques because of the influence of noise in the GPR amplitudes. Applying techniques such as regularization methods or filtering and stacking during data processing may help reduce the influence of noise in the conductivity analysis.

Whereas crosshole experiments have demonstrated the use of GPR for monitoring conductivity changes, there has been little work in applying the same technique to surface-based acquisition geometries. Here, we formulate the attenuation difference problem for multioffset GPR reflection data. Employing a number of simplifying assumptions, GPR attenuation difference tomography can be modeled and inverted like traditional slowness-based tomography. The total change in attenuation recorded at a receiver is the sum of attenuation changes along the raypath between the transmitter and the receiver. Instead of traditional grid-based tomographic inversions, we use adaptive-meshing theory to create a more even ray coverage among all of the model's cells. The objective of the adaptive meshing is to create an irregular mesh with every cell's resolution value between two user-defined thresholds. In addition to altering the inverse problem's operator matrix and model matrix, adaptive meshing based on cell resolution allows for the visual incorporation of resolution information into the final model. This new model can then be used with any tomographic inverse algorithm based on ray lengths per cell to compute the attenuation change within each cell.

Manuscript received by the Editor 31 July 2009; revised manuscript received 23 January 2010; published online 30 September 2010

${ }^{1}$ Boise State University, Boise, Idaho,U.S.A.E-mail: eahinz@cgiss.boisestate.edu; johnb@cgiss.boisestate.edu.

(C) 2010 Society of Exploration Geophysicists. All rights reserved. 
We investigate the adaptive meshing algorithm and reflection attenuation difference tomography using a simulated sand and cobble aquifer.

\section{ATTENUATION DIFFERENCE TOMOGRAPHY}

Intrinsic GPR attenuation is strongly influenced by the bulk conductivity of the medium the electromagnetic wave travels through. As discussed by Bradford (2007), the GPR frequency-dependant attenuation $\alpha$ can be approximated by

$$
\alpha=\frac{\sigma_{\mathrm{ef}}}{2} \sqrt{\frac{\mu}{\varepsilon^{\prime}}},
$$

where $\sigma_{\text {ef }}=\sigma^{\prime}+\omega \varepsilon^{\prime \prime}$.

The mathematical symbols are summarized in Table 1 , and the magnetic permeability is taken as $\mu=\mu_{0}$. Here we assume the imaginary term of the complex dielectric permittivity is approximately independent of frequency over the bandwidth of the signal. This is often reasonable in materials where GPR propagates effectively. Furthermore, we assume only the DC component of conductivity is changing with the addition of a saline tracer.

Table 1. Symbols for mathematical expressions.

\begin{tabular}{|c|c|c|}
\hline Symbol & Units & Description \\
\hline$a$ (Archie's) & & Fitting factor \\
\hline$A$ & & Amplitude \\
\hline$A_{0}$ & & Initial source signal amplitude \\
\hline$\alpha$ & $\mathrm{Np} / \mathrm{m}$ & Attenuation \\
\hline$C_{R}$ & & Receiver coupling factor \\
\hline$C_{S}$ & & Source coupling factor \\
\hline$\varepsilon_{\mathrm{ef}}$ & $\mathrm{F} / \mathrm{m}$ & $\begin{array}{l}\text { Effective dielectric } \\
\text { permittivity }\end{array}$ \\
\hline$\varepsilon^{\prime}$ & $\mathrm{F} / \mathrm{m}$ & Real dielectric permittivity \\
\hline$\varepsilon^{\prime \prime}$ & $\mathrm{F} / \mathrm{m}$ & $\begin{array}{l}\text { Imaginary dielectric } \\
\text { permittivity }\end{array}$ \\
\hline$\theta$ & & Porosity \\
\hline$G$ & $\mathrm{~Np} / \mathrm{m}$ & Geometric spreading \\
\hline$m$ (Archie's) & & Cementation exponent \\
\hline$\mu$ & $\mathrm{H} / \mathrm{m}$ & Magnetic permeability \\
\hline$N$ & $\mathrm{~m}$ & Total raypath distance \\
\hline$n$ (Archie's) & & Saturation exponent \\
\hline$\Theta_{S}$ & & Source radiation pattern \\
\hline$\Theta_{R}$ & & Receiver radiation pattern \\
\hline$R$ & & Reflection coefficient \\
\hline$\Gamma_{A}$ & & $\begin{array}{l}\text { Logarithmic amplitude } \\
\text { difference }\end{array}$ \\
\hline$r$ & $\mathrm{~m}$ & Ray length \\
\hline$\sigma$ & $\mathrm{S} / \mathrm{m}$ & Conductivity \\
\hline$\sigma_{\text {ef }}$ & $\mathrm{S} / \mathrm{m}$ & Effective conductivity \\
\hline$T$ & & $\begin{array}{l}\text { Transmission loss across } \\
\text { overburden layers }\end{array}$ \\
\hline$\omega$ & $1 / \mathrm{s}$ & Angular frequency \\
\hline$V$ & $\mathrm{~m} / \mathrm{s}$ & Velocity \\
\hline
\end{tabular}

The observed electric field amplitude measured by GPR is a combination of a variety of terms (Bradford and Deeds, 2006):

$$
A=A_{0} \frac{\Theta_{S} \Theta_{R} C_{S} C_{R} R T e^{-\alpha r}}{G} .
$$

The time-lapse amplitude difference (Johnson et al., 2007) caused by a conductivity change between two times, 1 and 2, is

$$
\Gamma_{A}=\ln \left(A_{1} / A_{2}\right)=\sum_{j=1}^{N} \Delta \alpha_{j} r_{j} .
$$

For the purposes of our study, this simplified form of expressing amplitude change produces an equation where attenuation change can easily be solved for with any number of inversion algorithms. The relatively simple relationship between attenuation and amplitude change relies on a number of assumptions, which can be briefly described as follows:

1) Changes in intrinsic attenuation are the main driver of attenuation differences implying that changes in reflection coefficients between the two times are negligible. Analysis of amplitude changes from FDTD modeling suggests scattering attenuation affects and reflection coefficient changes are at least an order of magnitude smaller than intrinsic attenuation changes (see the synthetic data discussion below).

2) Effective dielectric permittivity does not change through time and conductivity changes are small, therefore velocity changes between the two times are negligible. Velocity is given as

$$
V=\left(\frac{\varepsilon_{\mathrm{ef}} \mu}{2}\left(\sqrt{1+\left(\frac{\sigma_{\mathrm{ef}}}{\varepsilon_{\mathrm{ef}} \omega}\right)^{2}}+1\right)\right)^{-1 / 2}
$$

We expect effective conductivity changes due to the introduction of saline water in a typical tracer test to be on the order of tens of $\mu \mathrm{S} / \mathrm{cm}$. A change of $100 \mu \mathrm{S} / \mathrm{cm}$ would result in only a $0.5 \%$ velocity change.

3) Conductivity and dielectric permittivity are independent of frequency, and consequently dispersion is small. In the GPR frequency band this approximation is often reasonable and is often used in GPR analysis.

4) The grain surface conductivity effects are ignored, and the high-frequency relaxation properties are constant so that the only change between the models is DC water conductivity.

\section{RESOLUTION-BASED ADAPTIVE MESHING}

Our resolution-based adaptive meshing algorithm builds on ideas presented by Böhm et al. (2000) and Ajo-Franklin et al. (2006). An overview of the processing steps needed for attenuation-difference analysis is outlined in Figure 1. Because our inverse formulation only considers intrinsic attenuation, the velocity analysis and processing steps needed to create an appropriate velocity model and stacked section are separate from the process needed for the adaptive meshing and attenuation analysis. Additionally, because we assume that there are negligible velocity changes, we can use the same velocity model for all of the attenuation difference data sets. This also implies that raypaths only need to be calculated once for all of the data sets used to compare attenuation differences. The following subsections outline the process of creating the resolution-based mesh from a raw data set. 


\section{Horizon and amplitude picking}

Horizons used to trace raypaths through the model in the attenuation inversion are picked from stacked data. The stack may be generated via a standard NMO processing scheme or using time or depth prestack migration. We pick horizons that are strongly reflective and laterally coherent over a large lateral extent. They do not have to be limited to the extent of a single geologic feature nor do they have to be present throughout the entire section. We use the horizons to guide amplitude-picking in the multioffset, moveout corrected domain.

To eliminate the effect of phase variation in the measured amplitudes, we use the envelope function or instantaneous phase (Taner et al., 1979). We locate the local maxima of the envelope function in the vicinity of the picked horizon then sum the amplitudes over one wavelength symmetrically about the maxima. An imperfect moveout correction may result in small remnant residual moveout (RMO) that deviates from the picked horizon. We compensate for this RMO by using the local maxima from each successive trace as a starting point, thereby steering the horizon pick along RMO.

The integrated amplitudes at each horizon and offset are used as the amplitude $A$ in equation 3 for the attenuation analysis. All of the reflection locations are found in one data set, and the same location is used for all other attenuation difference data sets.

\section{Initial model construction and meshing}

Following the adaptive meshing concepts outlined by Böhm et al. (2000), we have chosen to use the dual relationships of Voronoi polygons and Delaunay triangles as the geometric foundation of our meshing algorithm. Both Voronoi polygons and Delaunay triangles are constructed using nearest neighbor properties and information, and a regular square grid is a special case of Voronoi polygons (Goodman and O'Rourke, 2004). The initial model is created by populating the model area with a set of points that are then associated with constrained Voronoi polygons and Delaunay triangles (Figure 2). Our algorithm starts with a Delaunay triangulated mesh for wavefront-based ray tracing and then converts the mesh to Voronoi polygons for adaptive meshing. While triangulated mesh offers an efficient data structure for calculating raypaths through the model, our adaptive-meshing algorithm is more easily implemented with Voronoi polygons.

Each point in the model area, also known as a Voronoi site, is enclosed by a unique Voronoi polygon and is also a vertex of a Delaunay triangle. Unconstrained Voronoi polygons are polygons whose edges are equidistant to the neighboring Voronoi sites. Constrained Voronoi polygons are clipped by secondary line segments, and, consequently, these polygons may violate the fundamental nearest-neighbor property of Voronoi polygons. For our adaptive-

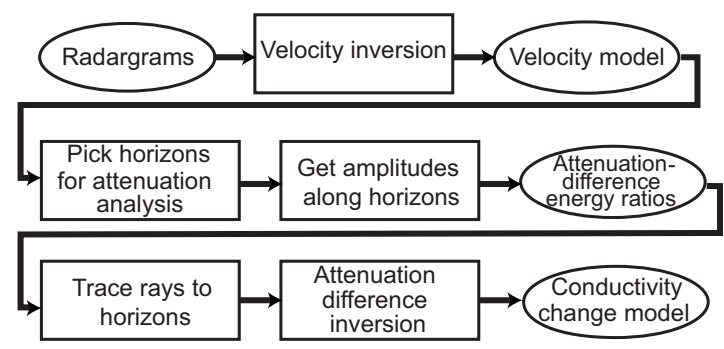

Figure 1. Outline of model creation and processing steps. meshing algorithm, the only constraints on the Voronoi polygons are the model area boundaries.

While both triangulations and Voronoi polygons have been used in previous mesh-adaptation studies (Böhm et al., 2000; Ajo-Franklin et al., 2006), the use of polygons instead of triangles for the adaptive meshing has two advantages. First, for a given set of points, the number of Voronoi polygons is about $\frac{1}{2}$ to $\frac{2}{3}$ the number of Delaunay triangles, and this will result in smaller matrices for the inversion algorithm. Second, the resolution-based mesh-altering algorithm that we define below is easier to implement with Voronoi polygons because deletion or addition of an entire polygon is simply the deletion of the polygon's unique Voronoi site or the addition of another Voronoi site within the polygon. The new model is created by remaking the Voronoi polygons. However, the adaptive-meshing algorithm we present could be rewritten for use on a triangulated mesh instead, by redefining the deletion or addition algorithm.

\section{Ray tracing}

Efficient and robust raypath computation is a critical component in the design of the ray-tracing algorithm. We accomplish this by first using a fast-marching eikonal equation for triangulated meshes (Kimmel and Sethian, 1998; Sethian and Vladimirsky, 2000; Rawlinson and Sambridge, 2004; de Kool et al., 2006) to calculate the wavefront arrival time from each source location through complex velocity fields (Rawlinson and Sambridge, 2004; de Kool et al., 2006). Raypaths are then traced along time gradients computed at each node of the triangular mesh to first order accuracy. The triangulated mesh ensures that nonhorizontal horizons can be modeled with the reflecting rays (Rüger and Hale, 2006). In addition, the accuracy of the computed time gradients and the resulting ray tracing is affected by the point sampling density of the velocity model which is a function of the coarseness of the triangulated mesh. We trace reflecting rays using a method based on the algorithm of Rawlinson and Sambridge (2004) which efficiently finds large sets of rays that share the same source point. For each unique source position, a synthetic wavefront is propagated through the entire model, and the traveltimes to every node are calculated and stored. The primary upgoing reflected wave is then computed from each reflecting horizon. For each horizon, the traveltime at each node along the horizon is used as
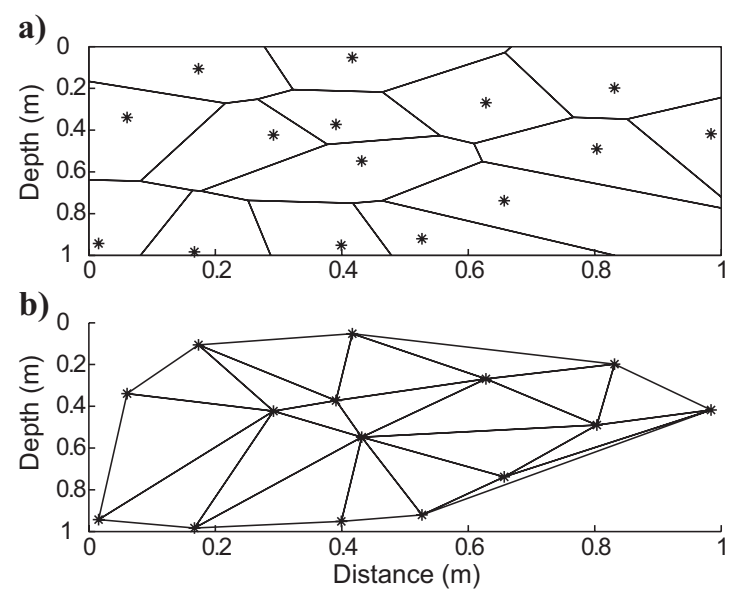

Figure 2. (a) Voronoi polygons constrained by the bounding rectangle and (b) dual Delaunay triangulation for a set of points. The points (asterisks) are both the vertices of the triangulation and the Voronoi sites. 
the initial conditions for a new fast-marching eikonal computation. The traveltimes are converted into time gradients through trilinear interpolation. Rays are then traced backwards through the time gradients, starting at the receiver and ending at the source. This shotpoint and Eikonal wavefront-based approach is an especially effective method for tracing rays in common-source gather (CSG) geometry, where the number of receiver locations is greater than the number of source locations.

\section{Resolution-based adaptive meshing}

We utilize adaptive meshing to redistribute model cells so that all of the cells possess resolution values between a user-defined minima and maxima. These criteria result in a model where areas with very high resolution contain more cells than areas with low resolution. The adaptive meshing algorithm we have implemented is based on Böhm et al. (2000) and Rüger and Hale (2006). The algorithm uses the diagonals of the resolution matrix derived from the singular-value decomposition (SVD) of the model matrix D:

$$
\mathbf{D}=\mathbf{U} \times \boldsymbol{\Lambda} \times \mathbf{V}^{\mathrm{T}} .
$$

$\mathbf{D}$ is an $\mathbf{M} \times \mathbf{N}$ matrix, where $\mathbf{M}$ is the number of rays and $\mathbf{N}$ is the number of cells that contain the ray length per cell. The decomposition results in three matrices, $\mathbf{U}, \boldsymbol{\Lambda}$, and $\mathbf{V}$, containing data-related eigenvectors, the square root of eigenvalues, and model-related eigenvectors, respectively. The resolution matrix $\mathbf{R}$ is calculated as

$$
\mathbf{R}=\mathbf{V} \times \mathbf{V}^{\mathrm{T}}
$$

and ranges from zero to one, where zero is a cell with no intersecting rays and one is a perfectly resolved cell. The diagonal of the resolution matrix, which we will term the fitness vector, contains information on the resolution of each polygon. In addition, we include a minimum segment-length criterion to prevent infinitely thin cells along the raypath. This ray-length criteria is problem-specific, but factors such as the GPR wavelength resolution and the positioning accuracy of the ray-tracing algorithm should be incorporated into the choice of the minimum segment length. We find that the minimum segment length should be at least the vertical resolution of the GPR system, $\frac{1}{4}$ of the wavelength, to approximate the accuracy of locating the picked horizons. All cells are required to contain at least one ray segment with a ray length greater than the minimum segment length criteria. The choice of the minimum required ray-segment length is important to keep the polygons larger than the wavelength used to calculate the amplitude changes, and within the accuracy of the raytracing algorithm to correctly position raypaths in the model.

Computing the SVD of a matrix becomes increasingly time consuming and problematic for very large matrices that are typical for tomographic problems, and the calculation can become a bottleneck in the adaptive-meshing routine. Instead of calculating the SVD of matrix D, we approximate the SVD through a truncated Lanczos bidiagonalization algorithm developed by Larsen (1998) for the Pavtial Reorthogonalization Package (PROPACK). Because the resolution matrix is used as a guide to split or merge Voronoi polygons and not for a full inversion of the data, the adaptive-meshing algorithm can use an approximation to the full SVD. We arbitrarily keep the $66 \%$ of the largest eigenvalues to approximate a truncated SVD. Truncation increases the speed of calculating the resolution matrix, and it also results in more stable decompositions. Zhang and Thurber (2007) give a more in-depth discussion on the use of Lanczos bidiagonalization for approximating the SVD and resolution matrices.
Two thresholds, a polygon-splitting and polygon-merging threshold, are defined by the user. The merging threshold is a minimum allowable fitness value whereas the splitting threshold is a maximum fitness value. Polygons with fitness values below the merging threshold or that do not contain a ray segment above a minimum segment length are deleted. In contrast, polygons with fitness values above the splitting threshold are split into two separate polygons. When polygons are split, we sacrifice some of the ray density and model resolution for a smaller cell. An extreme example of this case would be a model covered by a single cell. While this cell has a fitness value of one, the model has averaged all of the spatial information into a single cell. Because each polygon is defined by a single unique Voronoi site, the merging and splitting routines can operate on the set of Voronoi sites, and a new Voronoi polygon set is computed from the altered point set. Each time a new Voronoi polygon set is constructed, a new resolution matrix is calculated for the updated model. The adaptive-meshing algorithm iterates until all of the polygons possess fitness values that lie within the acceptable fitness threshold or until a user-defined number of iterations are performed.

The merging and splitting thresholds are problem specific, and picking appropriate values often involves some experimentation to evaluate the effect of different ranges on the model space. The merging and splitting thresholds also greatly affect the size of the final model, and consequently, the thresholds directly affect the computational expense in calculating an inverse solution. A narrower splitting and merging range will result in a more even distribution of rays and eigenvalues than a broader range, but at the same time, a narrower range will result in reshaping more cells and larger computations than a broader range. In general, we recommend a conservative splitting threshold between 0.8 and 0.9 and a merging threshold between 0.4 and 0.6. These criteria result in a new model space with an adequate compromise between high fitness values, a relatively narrow fitness range, and computation time.

\section{Attenuation difference inversion}

The simultaneous iterative reconstructive tomography (SIRT) algorithm provides a simple approximation to a least-squares inversion to solve for the attenuation change per Voronoi polygon. Raylength averaged updates to the attenuation are computed and applied once per iteration. We implement the parameter update weighting method presented in Aster et al. (2005), which weights based on the number of ray segments per cell and ray length. A major drawback of using an irregular mesh for the model space instead of a regular grid is that regularization techniques that utilize spatial derivatives are difficult and computationally costly to implement. Many spatiallybased regularization techniques, such as L2-normalization, are defined only for rectangular grids. Our inversion uses no additional constraints on the range of attenuation values or smoothing regularization. The attenuation change is converted into effective conductivity change by solving for conductivity using equations 1 and 3 .

\section{SYNTHETIC AQUIFER EXAMPLE}

\section{Wavefield simulator}

We use a finite-difference time-domain (FDTD) algorithm to create synthetic GPR common source gathers. The FDTD algorithm uses standard second-order accurate finite-difference operators (Levander, 1989) to solve the two-dimensional, isotropic, second-order electric-field wave equation with nonzero conductivity. An absorb- 
ing boundary condition is implemented by introducing the conductive region around the model space. We simulate TE polarization (field perpendicular to model plane) and introduce a profile perpendicular line source at the surface of the model. For all simulations below, we use a $100 \mathrm{MHz}$ Ricker wavelet as the source. Because we are inverting for attenuation changes and we will assume that there is no attenuation or dielectric permittivity change near the antenna positions, we can ignore antenna directionality.

\section{Forward modeling}

The aim of our synthetic aquifer example is to simulate a saline tracer injected at depth into a permeable sand unit. We produce a model of the subsurface with realistic heterogeneity by incorporating velocity and conductivity heterogeneities derived from a modified photograph of a sand-gravel outcrop (Figure 3). This outcrop is an analog of the subsurface materials and properties at the Boise Hydrogeophysical Research Site (BHRS), located $2.5 \mathrm{~km}$ downstream of the outcrop. The BHRS is a research well field southeast of Boise, Idaho, underlain by an approximately 20 -m-thick sequence of unconsolidated sandy pebble- and cobble-dominated fluvial sequences (Barrash and Reboulet, 2004). While the synthetic aquifer example is not designed to perfectly simulate the geology and stratigraphy beneath the BHRS, the photo-derived properties simulate realistic heterogeneous distributions of petrophysical properties.

To produce a subsurface model, the photograph is converted to gray scale, and the pixel values are then interpolated to porosity by assigning porosities that range from zero for black cells to one for white cells. Visual inspection of the outcrop reveals that light colors in the photograph correspond with sandy units while dark colors correspond with cobble units. Porosity values are clipped to a maximum of 0.5 to prevent unrealistically high porosities, and the high resolution photograph is resampled to a pixel size equivalent to the spatial step size of the FDTD algorithm (Figure 4a). A comparison of the porosity values from different units of our synthetic outcrop model and the overall porosity distribution is shown in Figure 5.

We introduce a shallow water table into the porosity model at $1.5 \mathrm{~m}$. We assign a pore water saturation of $18 \%$, due to residual water content, within the vadose zone, and the pores are assumed to be fully saturated below the water table. The vadose zone pore water saturation is analogous to values observed at the BHRS. Since our conductivity change anomaly will be contained within the fully saturated zone, varying the choice of residual water saturation in the vadose zone or the nature of the saturated-unsaturated zone contact will only slightly affect the raypaths traced through the model and will not significantly affect the final attenuation inversion. We also

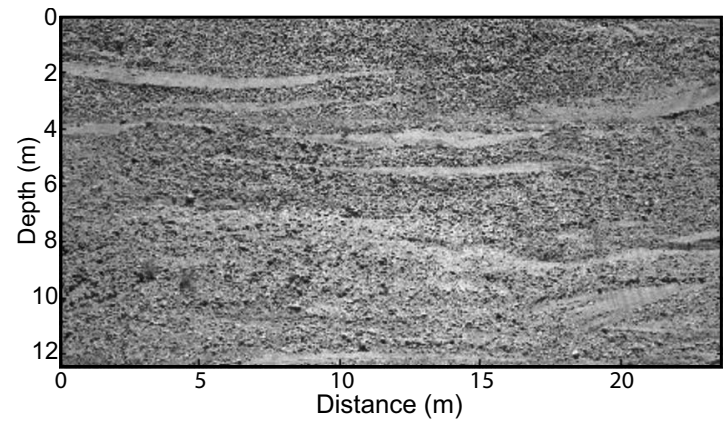

Figure 3. Enhanced grayscale photograph of a sand-cobble outcrop used to construct the BHRS analog synthetic models. assume the matrix material is composed of only sand grains, and therefore we can use Archie's Law and a three phase complex refractive index method (CRIM) equation to approximate the effective conductivity and dielectric permittivity, respectively (Figure $4 \mathrm{~b}$ and c).

Archie's Law is an empirical formula that relates the effective conductivity of a clean sandstone sample to the effective conductivity of the pore water (Knight and Endres, 2005). The equation assumes a resistive matrix so that the majority of a sample's conductivity is from the pore water $w$ :

$$
\sigma=\frac{1}{a} \sigma_{w} \theta^{m} S_{w}^{n}
$$

The dielectric permittivity, and hence velocity, is estimated by mixing the matrix and fluid conductivities using the CRIM equation (Wharton et al., 1980). For our three-phase system (solid matrix $m$, water $w$, and air $a$ ), the CRIM equation can be written as
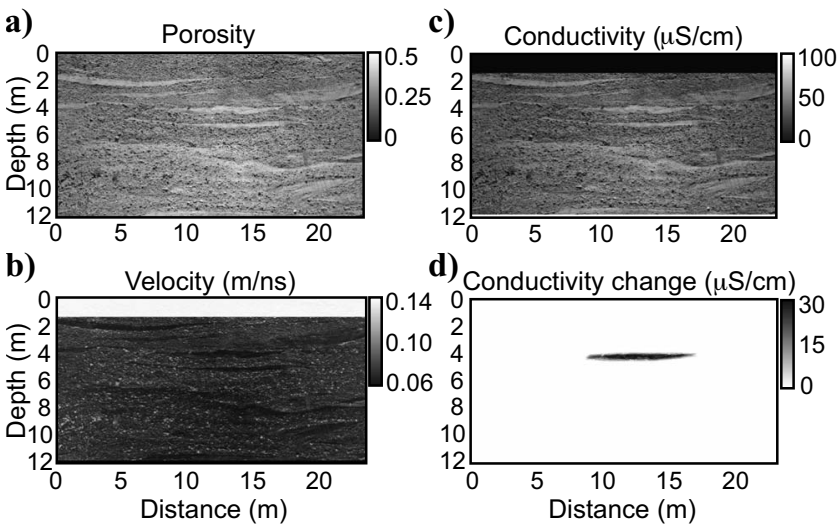

Figure 4. (a) Porosity, (b) velocity, and (c) conductivity values for the background model derived from Figure 3. (d) Conductivity change from increasing the water conductivity by $100 \mu \mathrm{S} / \mathrm{cm}$ within one sand unit. The water table is at $1.5-\mathrm{m}$ depth and can be seen as a sharp discontinuity in both the derived velocity and conductivity models (b and c).
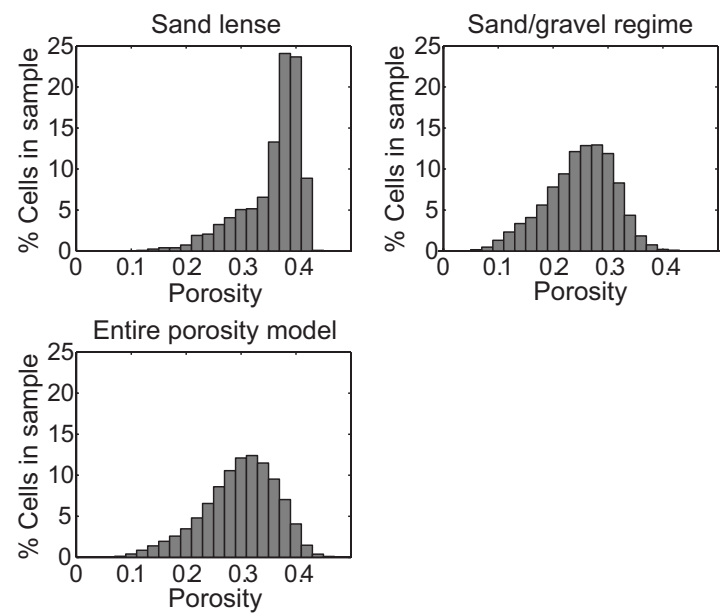

Figure 5. Porosity distribution from the synthetic model shown in Figure $4 a$. 


$$
\sqrt{\varepsilon}=(1-\theta) \sqrt{\varepsilon_{m}}+\theta S_{w} \sqrt{\varepsilon_{w}}+\theta\left(1-S_{w}\right) \sqrt{\varepsilon_{a}} .
$$

Other parameters used to create the surface-based synthetic radargrams are described in Table 2.

The conductivity change is modeled by increasing water conductivity by $100 \mu \mathrm{S} / \mathrm{cm}$ within the $\sim 0.5$-m-thick sand lense centered at 4.5-m depth between 9- and 17-m distance, and this water conductivity change results in a bulk conductivity change of approximately $30 \mu \mathrm{S} / \mathrm{cm}$ (Figure 4d). Water conductivity values are smoothed around the edges of the sand lense to simulate slight dispersion of the conductive water. Additionally, a flat clay layer is appended to the bottom of the model to give a strong coherent reflector that allows us to check the quality of our velocity model and migration.

Amplitude-repeatability tests at the BHRS with a Sensors and Software PE100A using 100-MHz antennas indicate that logarithmic real enveloped amplitude difference $\mathrm{R}_{A}$ typically vary by as much as 0.05 . The change in conductivity used in our synthetic aquifer example creates amplitude differences at their maximum of $0.2-0.3$, or approximately an order of magnitude larger than the noise level of real GPR data. Uniformly distributed random noise between -0.05 to 0.05 were added to the logarithmic amplitude differences to simulate the expected noise level.

\section{Preprocessing}

As implemented by Stork (1992), we use a reflection tomograph in the prestack depth migration (PSDM) domain to estimate the velocity model. In this method, traces are prestack depth migrated with an initial velocity model, and reflectors are then checked to see if they are flat-lying in the common-imaging-point (CIP) domain. An incorrect velocity model will result in reflectors that are not flat, and the offset-dependent change in the reflector is the residual moveout. The velocity model is updated to remove residual moveout, and the data are then remigrated. This process is repeated until the RMO analysis converges on a velocity model. Bradford $(2006,2008)$ and Bradford et al. (2009) provide a more detailed discussion of reflec-

Table 2. Property values used to generate the surface-based synthetic aquifer data.

\begin{tabular}{llc} 
Property & \multicolumn{1}{c}{ Phase } & Value \\
\hline Dielectric constant & Sand matrix & 4.7 \\
Dielectric constant & Water & 81 \\
Dielectric constant & Air & 1 \\
Conductivity $(\mu \mathrm{S} / \mathrm{cm})$ & Sand matrix & 0 \\
Background model conductivity $(\mu \mathrm{S} / \mathrm{cm})$ & Water & 220 \\
Saline tracer conductivity $(\mu \mathrm{S} / \mathrm{cm})$ & Water & 320 \\
$a$ (Archie's) & & 1 \\
$n$ (Archie's) & & 2 \\
$m$ (Archie's) & & 1.3 \\
FDTD spatial cell width and height $(\mathrm{m})$ & & 0.0244 \\
FDTD time step $(\mathrm{ns})$ & & 0.1251 \\
Resampled time step $(\mathrm{ns})$ & & 0.8 \\
Total time $(\mathrm{ns})$ & & 500 \\
Source spacing $(\mathrm{m})$ & & 1 \\
Receiver spacing $(\mathrm{m})$ & & 0.2
\end{tabular}

tion tomography analysis with GPR data. The resulting velocity function from the reflection tomography analysis is able to reproduce large-scale velocity variations of the original model (Figure 6). Velocity estimates along the edge of the migrated stack are less wellconstrained because these regions have less fold to constrain the velocity inversion process.

We picked four horizons from the PSDM stack: two horizons defining the top and bottom of the sand lens, and an additional horizon above and below the sand lens (Figure 7). The amplitudes along each of the picked horizons are used with equation 3 to compute the amplitude ratio $\mathrm{R}_{A}$. With our aquifer model, we were able to confidently pick the top of the sand unit, but the sand lens quickly thins below the $100-\mathrm{MHz}$ wavelet resolution limit making it difficult to accurately pick the entire length of the bottom of the sand unit. Small inaccuracies in the location of picked horizons are a result of the finite bandwidth of the 100-MHz data and inaccuracies in our estimated velocity model. The velocity model produced through the RMO velocity analysis procedure overestimates areas of low velocity and underestimates areas of high velocity by about $0.01-0.02 \mathrm{~m} / \mathrm{ns}$ (Figure 6 ).

a) 0

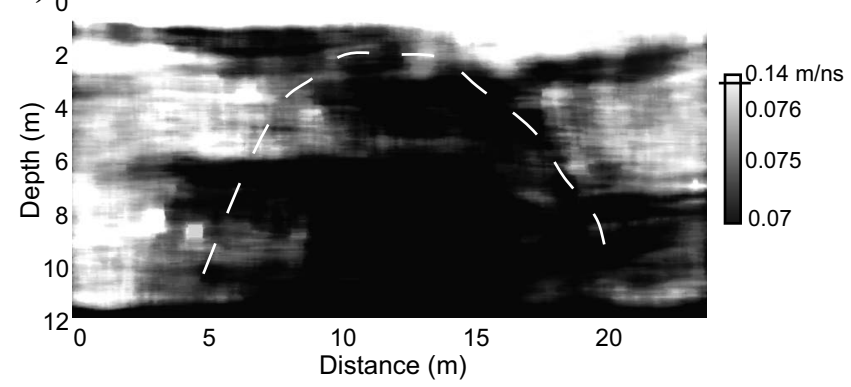

b) 0

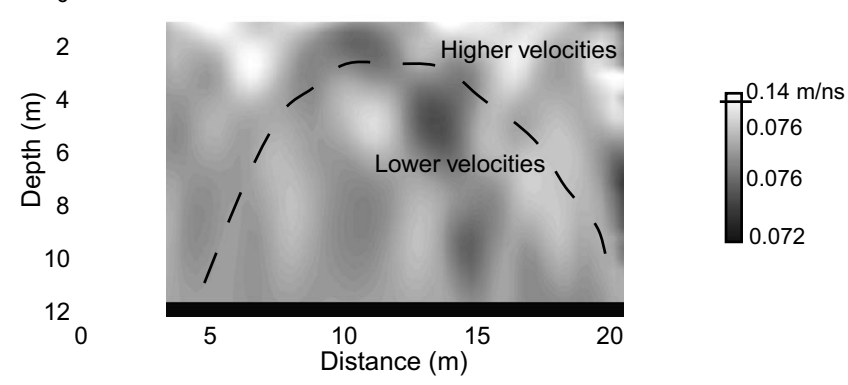

c) 0

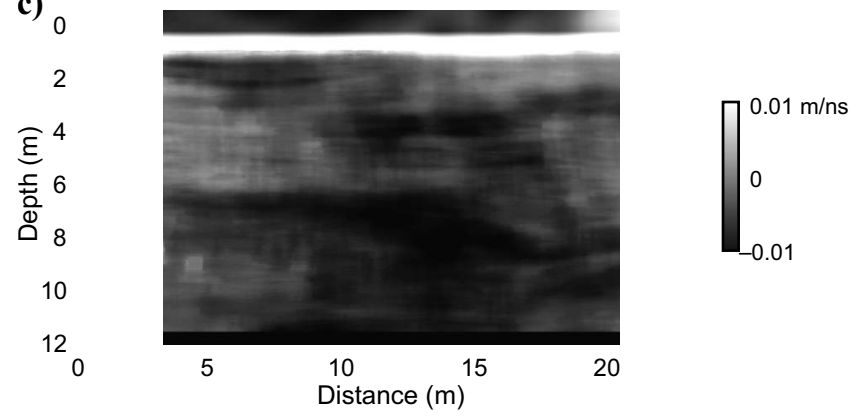

Figure 6. (a) The original velocity function shown in Figure 4b with a $1 \times 1 \mathrm{~m}$ running median smoothing operator applied. (b) Velocity field created from reflection tomography inversion. The velocity field is truncated to the range with good RMO velocity control, and the color scale is clipped more severely than for (a) to better highlight regions of velocity changes. (c) The velocity difference between (b) and (a). 
Over the region of the conductivity change, the velocity is overestimated resulting in migration horizons in this region that appear deeper than their true position. The horizon is at the peak amplitude of the reflected wavelet in the migrated depth-domain data, whereas the true location of the reflector is better represented by the first break.

\section{Results}

The initial model grid consists of 1600 0.5-m square cells through which 5113 reflecting rays were traced to the four picked horizons (Figure 7). As with most tomographic problems, the synthetic model is overdetermined; the number of observations, in this case the number of rays and corresponding amplitude changes, is much greater than the number of model cells. Only source, receiver, and horizon combinations that result in finding a local amplitude maximum within a single wavelength in the envelope trace function are used to trace rays. Some of the picks along the horizons, such as along horizon 4 (Figure 7b), exhibit small negative amplitude ratio changes, approximately $5 \%$ of the maximum computed amplitude ratio, before the addition of noise. Negative changes result from small scattering attenuation effects, but these negative changes are approximately an order of magnitude smaller than the intrinsic attenuationdifference effects.

Five iterations of the adaptive-meshing algorithm with fitness values constrained between 0.8 and 0.6 and a minimum ray segment length of $0.25 \mathrm{~m}$ were applied to a $0.5-\mathrm{m}$ square grid starting model. The resulting adaptively meshed (AM) model consists of 1596 polygons for the surface-reflection tomography example (Figure 8). After five iterations of the adaptive-meshing algorithm our algorithm had not yet found the optimal distribution of cells, and the AM model contains some cells that violate the adaptive-meshing criteria. However, because remeshing is a computationally intensive process for
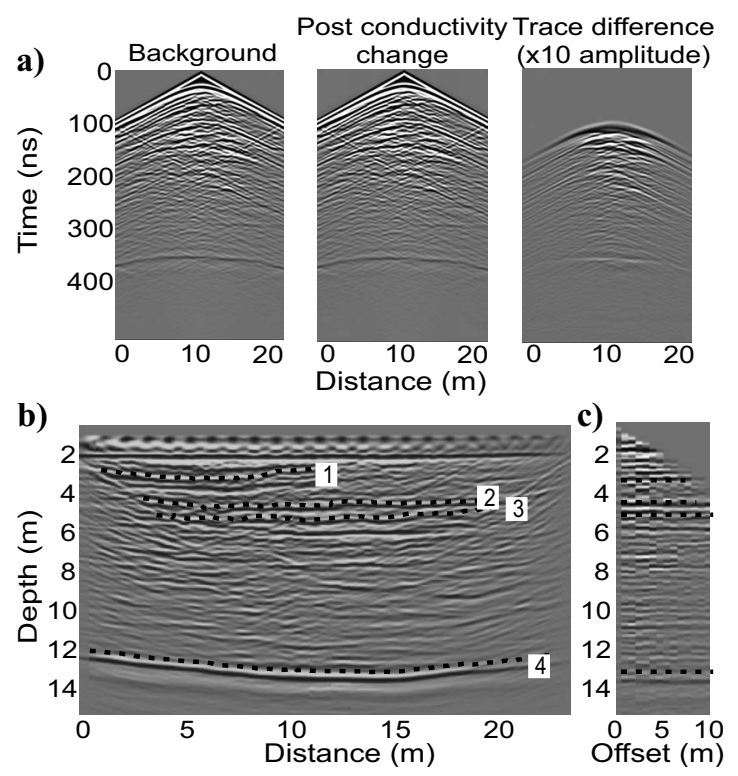

Figure 7. (a) Unprocessed synthetic shot gathers derived from the synthetic aquifer background and post-conductivity change models. The difference between the two gathers is shown with amplitudes magnified by a factor of 10. (b) The stacked and migrated section for the background model and (c) a common-imaging-point gather showing the location of the picked horizons. our large model, five iterations are sufficient to gain some of the adaptive-meshing algorithm's benefits in inversion efficiency and resolution distribution.

The picked reflecting horizons can be used to further constrain the inversion model. Clipping the AM model polygons by the picked horizons results in 1800 polygons. We will refer to this horizon-constrained variation on the adaptively meshed model as the AMH model. Some of the polygons in the AMH model, however, violate the minimum segment length or the fitness criteria. Additionally, the clipped polygons are no longer true Voronoi polygons. A more sophisticated adaptation algorithm that incorporates horizons as line constraints into the adaptive meshing process that would allow for all of the polygons to still adhere to the minimum segment length and fitness criteria, but this is beyond the scope of this paper.

The conductivity change inversion was performed on the original grid and the AM and AMH models. The SIRT inversion found a model with a minimum error 100 iterations sooner, or about $25 \%$ of the number of iterations, for the adaptive-mesh model than for the original square grid. Likewise, the decrease in iterations and the increase in the number of polygons is also reflected in the total CPU time and memory used in the inversion (Table 3). The derived conductivity model produced by the SIRT inversion for the original grid, $\mathrm{AM}$, and AMH models locate a large $(>10 \mu \mathrm{S} / \mathrm{cm})$ positive conductivity change between 10.5 - and 18.5 - $\mathrm{m} x$-distance and a depth between 4 and $5 \mathrm{~m}$ (Figure 9). The AMH model incorporates the raytracing horizons and gives a better visualization as to where the amplitude data originates.

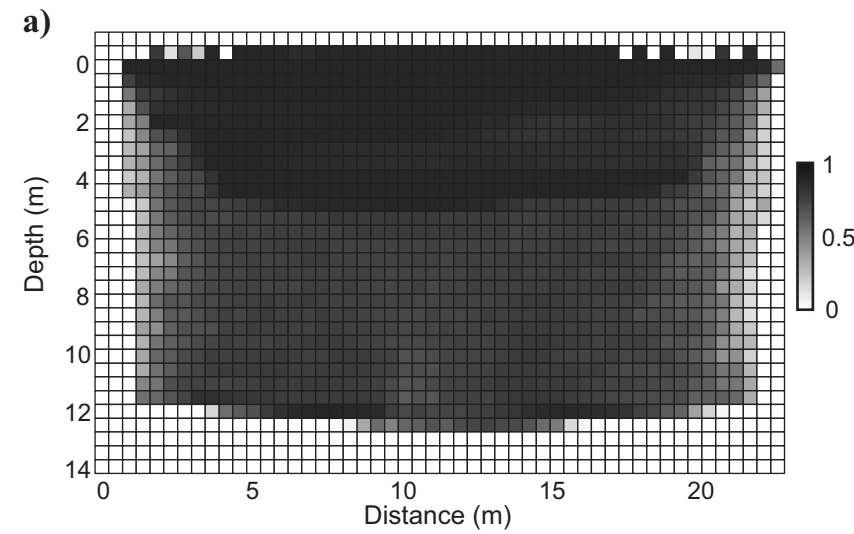

b)

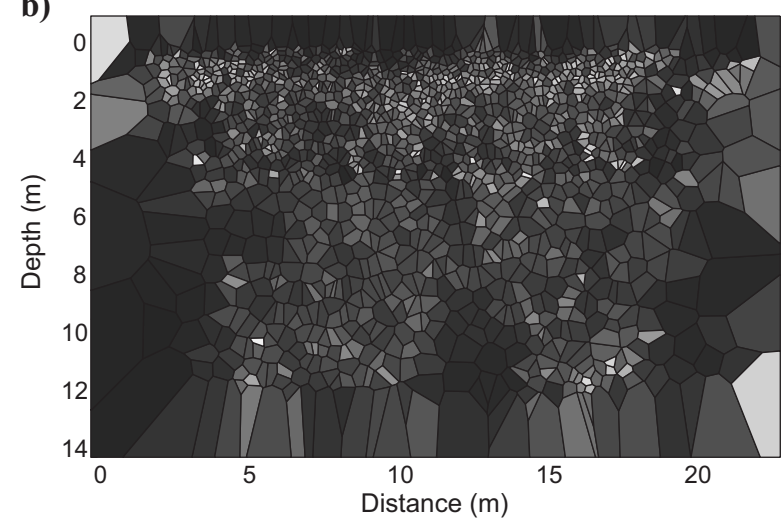

Figure 8. Fitness (resolution) of the polygons calculated from (a) a rectangular grid and (b) after adaptive meshing for the surface-reflection survey geometry. 
All of the inverted conductivity models from the surface-based reflection models, shown in Figure 9, underestimate the conductivity change. Furthermore, some cells contain negative conductivity changes as a result of some negative amplitude changes between the presaline and postsaline injection models (Figure 9). The inversion models also all incorrectly place a small area of conductivity change

Table 3. Computer performance metrics for the surface-reflection SIRT conductivity inversions.

\begin{tabular}{lcc} 
& CPU Time $(\mathrm{s})$ & Relative Memory Usage \\
\hline Grid & 550 & 1 \\
AM & 170 & 1.07 \\
AMH & 120 & 1.27 \\
\hline
\end{tabular}
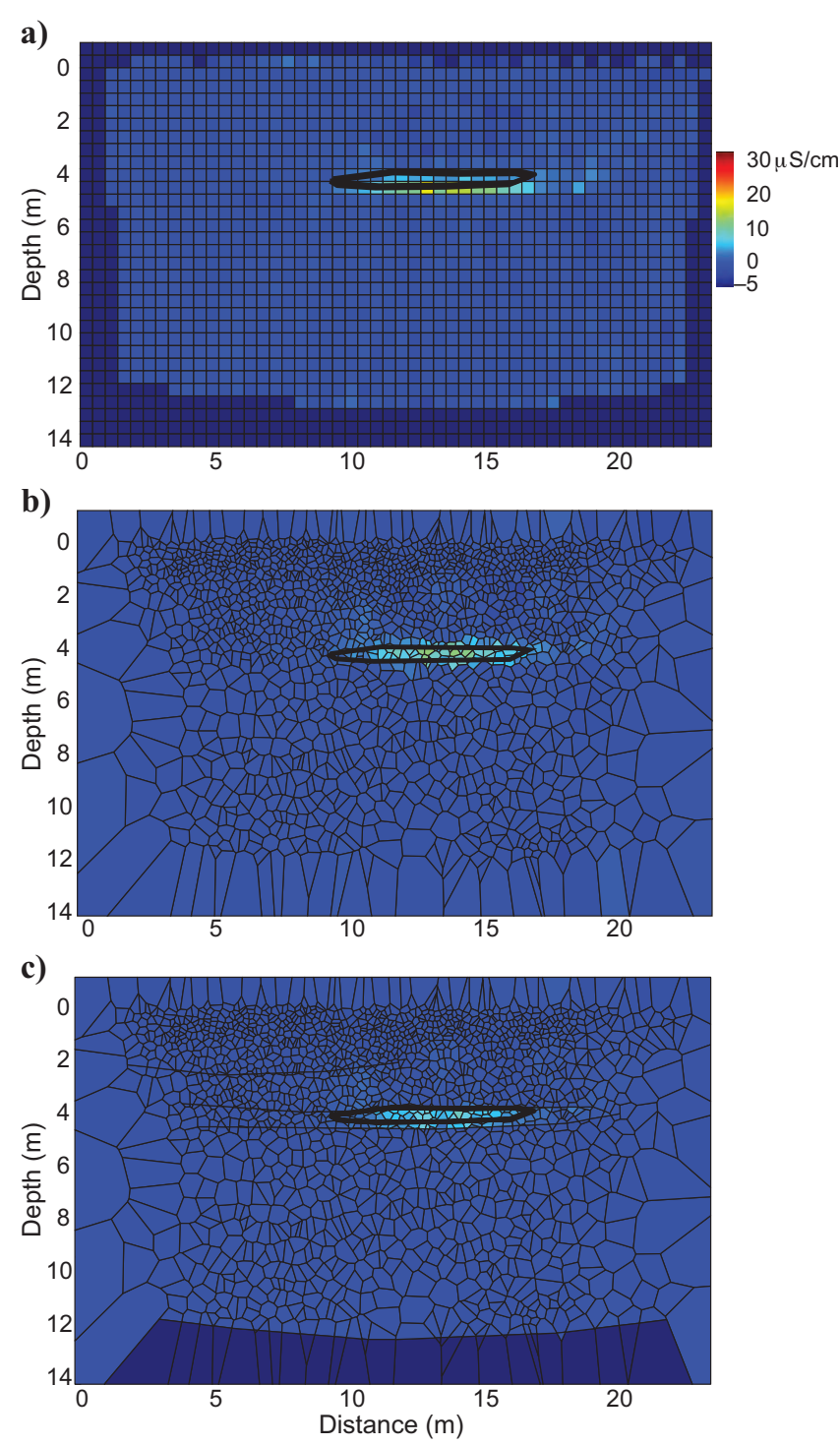

Figure 9. Estimated DC conductivity values computed from the attenuation inversion with (a) the grid, (b) the AM, and (c) the AMH models. The region of conductivity change is outlined in all three models. with a magnitude of about $5 \mu \mathrm{S} / \mathrm{cm}$ around a distance of $17 \mathrm{~m}$. This spurious region is related to a failure in amplitude summing and differencing along the picked horizons.

We calculate the misfit and the rms error between the original noise-free conductivity difference model (Figure 4d) and the tomographic models (Figure 9) by interpolating the tomographic models back onto the original grid. Comparing the inversion results using the surface geometry with respect to the original noise-free model, the clipped adaptively meshed model has the smallest rms error $(0.0028 \mu \mathrm{S} / \mathrm{cm}$ per cell $)$, followed by the adaptively meshed model $(0.0029 \mu \mathrm{S} / \mathrm{cm}$ per cell $)$ and then the $0.5-\mathrm{m}$ square grid $(0.0033 \mu \mathrm{S} / \mathrm{cm}$ per cell). Thus, for our example, adaptive meshing provides a $12-15 \%$ improvement over the regular grid.

\section{Comparison to crosshole geometry}

For comparison, we also simulate a crosshole radar experiment with the same synthetic data set. We place a source at 0.5 -m intervals with receivers at $0.2-\mathrm{m}$ intervals. These source and receiver spacing are coarser than typically found in borehole radar surveys, but this geometry yields a comparable number of rays to the surface-reflection tomography data set. Boreholes are located at an $x$-distance of $5 \mathrm{~m}$ and $21 \mathrm{~m}$ and span the entire depth of the model area. Sources and receivers were placed in both wells, and only direct wave energy was picked for use in calculating amplitude differences.

Instead of reflection events, the crosshole model uses first arrival energy, comprised of both refracted and direct energy for the attenuation difference tomography. A total of 4107 rays were traced through the initial model. After applying the same adaptive-meshing criteria used with the reflection model, the model contained 924 polygons (Figure 10). The region of poorer resolution and consequently larger cells in the adaptively meshed model between 2 - and $5-\mathrm{m}$ depth is a result of refracted events arriving before the direct wave for some of the source and receiver combinations.

The crosshole conductivity inversion places the region of conductivity change over a broader area than the surface reflection inversion (Figure 11). The spatial extent, both lateral and vertical, of the region of conductivity change is also poorly constrained by the crosshole inversion. The poorer conductivity change estimates are a result of the crosshole raypath geometry. As shown by the fitness estimates in Figure 10a, the region of the conductivity change has the poorest ray coverage and resolution in the model space. Not only does this result in a poor lateral and vertical constraint on the shape of the region of conductivity change, but the poor ray coverage also smears the conductivity change over the length of the raypaths that do image the conductivity change anomaly. An example of this smearing is illustrated in Figure 11a and $\mathrm{b}$ as small positive conductivity changes between a distance of 7 and $8 \mathrm{~m}$ at a depth of $8 \mathrm{~m}$.

Unlike the surface reflection model, the crosshole model does not show any improvement in rms error in the model area between the two wells; both the square grid and AM model have an rms error of $0.0032 \mu \mathrm{S} / \mathrm{cm}$ per cell. However, the SIRT inversion still found a minimum error model approximately 100 iterations sooner for the AM model.

\section{DISCUSSION}

The distribution and size of polygons in the adaptively meshed model provides information on the ray density through the model and also increases the spatial resolution in certain areas of the model. Since the densest ray coverage occurs near the surface of the model, 
the shallowest portions of the model have the highest fitness values in the original grid, and a majority of the polygons in the AM model cluster near the surface (Figure 8). As a result, polygons in areas with dense ray coverage and fitness values larger than the splitting threshold are smaller than the original $0.5 \mathrm{~m} \times 0.5 \mathrm{~m}$ cells. In these areas, the AM model can better resolve the spatial distribution of conductivity anomalies. However, in regions where ray coverage is sparse and the fitness values of the cells are smaller than the merging threshold, the polygons in the AM model are larger than the original grid. The shape of conductivity anomalies in these areas will appear less well-resolved in the AM model, but the low resolution in these areas of the original grid would result in a poor inverse solution.

Besides the resolution matrix, another method of evaluating the quality of the model space is to look at the resulting eigenvalues. Ideally, the squared eigenvalues should all be equal, creating a flat eigenspectrum. For instance, after adaptive meshing, the truncated eigenspectrum is flatter for the synthetic aquifer example (Figure 12). Applying more iterations and narrowing the merging and splitting thresholds used in the adaptive-meshing algorithm would result in greater flattening of the spectrum. However, the SVD resolutionbased adaptive-meshing algorithm that we implement requires solving for the operator matrix at each iteration of the remeshing algorithm, and this is a computationally intensive operation. Deriving different remeshing criteria independent of large matrix calculations associated with the SVD would greatly speed up the adaptive-meshing algorithm. Additionally, different criteria will affect the final model and the conductivity inversion. Exploration of the impact of
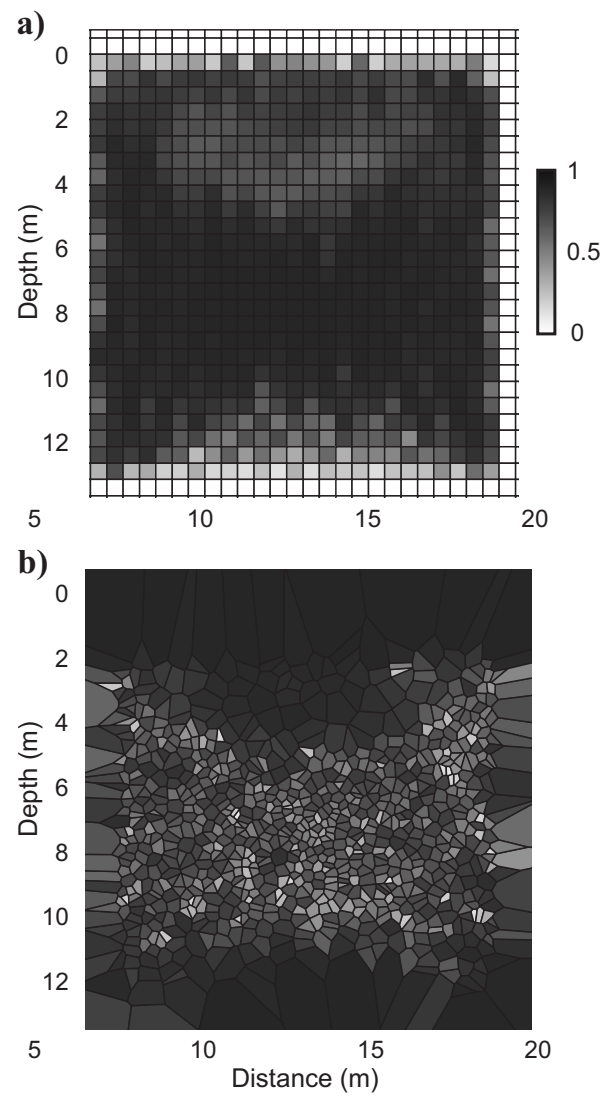

Figure 10. Fitness (resolution) of the polygons calculated from (a) a rectangular grid and (b) after adaptive meshing for the crosshole survey geometry. remeshing techniques on the inversion could yield improved adaptive-meshing techniques.

Because our adaptive-meshing algorithm does not incorporate geometric constraints, the adaptively meshed polygons are not constrained by any of the picked reflecting horizons. However, including the reflecting horizons in the new data set could increase the accuracy of the conductivity inversion. This is especially true in our two examples where the conductivity anomaly is confined within a layer that is also used as a reflecting horizon.

For our example, the multioffset reflection data creates a better estimate and spatial representation of the region of conductivity change than the comparable crosshole data. Because the area of con-
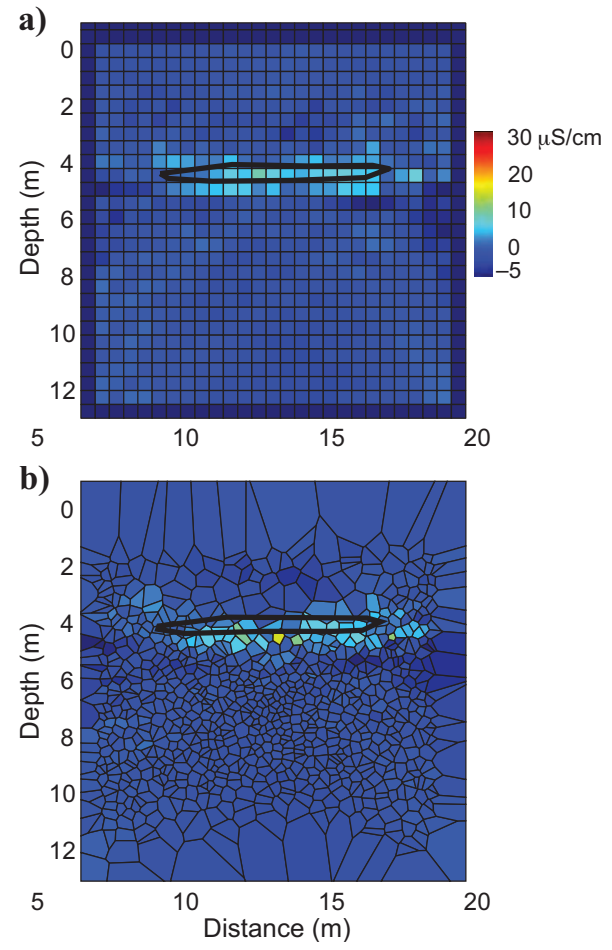

Figure 11. Estimated DC conductivity values computed from crosshole direct wave attenuation inversion with (a) the grid and (b) the AM models.

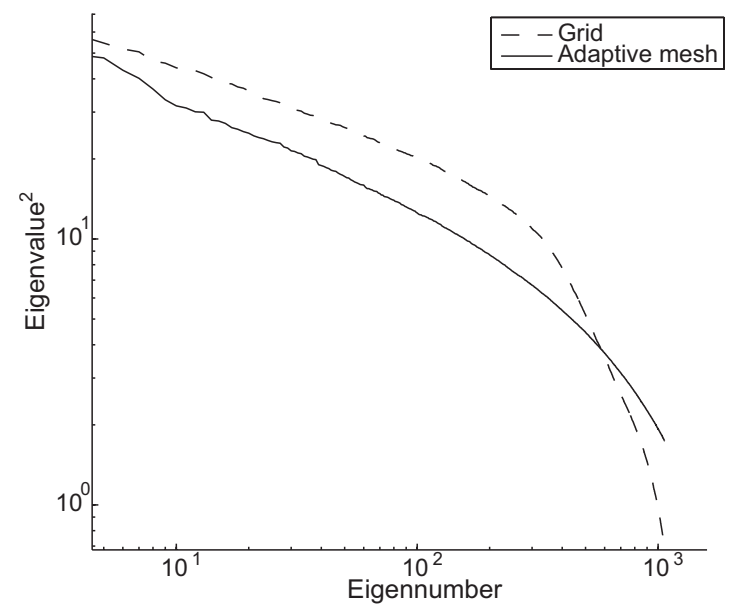

Figure 12. Spectrum of truncated eigenvalues for the grid and AM models. 
a)

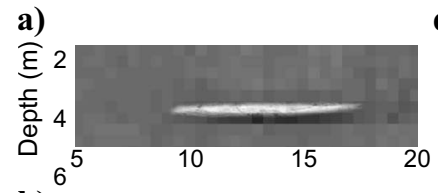

b)

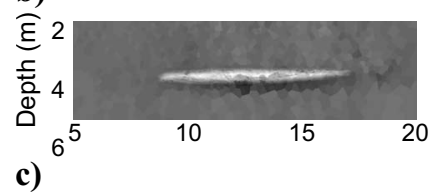

e)

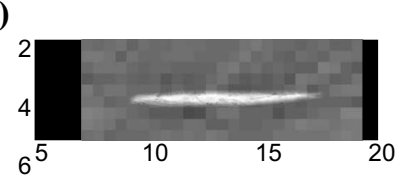

.

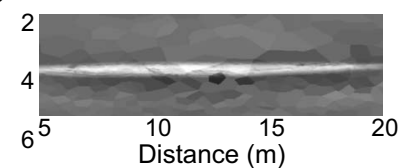

c)

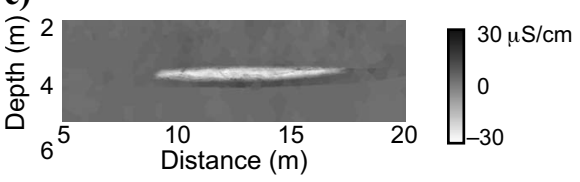

Figure 13. Model differences between the true conductivity change (Figure 4c), and the conductivity estimates derived from the surface reflection (a) grid, (b) AM, and (c) AMH models and the crosshole (d) grid and (e) AM models.

ductivity change is located near the water table, a large number of crosshole first arrivals are rays that refract through the higher-velocity vadose zone rather than arrive as energy traveling directly through the conductivity anomaly. Furthermore, the reflecting horizons used with the reflection data inversion constrain the vertical extent of the conductivity change.

Picking ray-tracing horizons that closely constrain the top and bottom of the region of conductivity change helps to constrain the model vertically. The good vertical resolution of the conductivity change from surface-based reflection tomography is a result of the conductivity change being confined in the model by strong reflectors. The reflectors allow us to pick the top and bottom of the conductivity change as reflecting horizons. The lateral resolution of the conductivity anomaly is limited by the source spacing because the zero and near-zero offset traces provide the best lateral constraints for the inversion.

All of the SIRT inversion results underestimate the DC conductivity within the plume (Figure 13). This is partly a result of some uncertainty in picking identical reflectors for amplitude comparison, and the noise added to the picked amplitudes but underestimation is mainly a result of the tomographic inversion process. This phenomenon associated with ray-based tomography has been observed in tomographic inversion and is the impetus for developing new methods of inversion (Böhm et al., 2000; Lane et al., 2004; Johnson et al., 2007). Tomographic inversion averages the attenuation change along the length of the raypath, and this can result in smearing the attenuation change over a larger region and along the raypath. Smearing is particularly evident in the crosshole conductivity change estimates (Figure 11).

\section{CONCLUSIONS}

Unlike crosshole GPR attenuation tomography, the quality of surface-based reflection attenuation tomography depends on the number and distribution of reflectors and on the accuracy of identifying the location of those reflectors. For instance, inaccuracies in the velocity model used for migration propagate into the final conductivity inversion. Furthermore, while reflection tomography offers the ability to both laterally and vertically resolve conductivity anomalies, the vertical extent of the region of conductivity change will be less

well resolved unless the anomaly is confined between well resolved reflectors. Vertical resolution can be improved by incorporating far offsets into the attenuation inversion and by choosing horizons close to the area of expected conductivity change. Likewise, the lateral resolution of the shape of the conductivity anomaly is dependent on the geometry of data acquisition. Source spacing affects the density of near-offset data that provide spatial constraints on the lateral extent of conductivity change. Large offsets can also improve lateral and vertical resolution, especially for conductivity anomalies that span multiple reflectors.

Adaptive meshing based on the resolution matrix of the model space creates a new model space with a more even distribution of model resolution and a flatter eigenvalue spectrum. By using the resolution matrix to steer the adaptive meshing, we use the distribution of data to drive the creation of our new model space. The new model space concentrates model polygons where the ray density and resolution is at its greatest and reduces the number of model polygons where the resolution is small or rays are nonexistent.

The redistribution of rays per polygon by the adaptive-meshing algorithm results in a faster SIRT inversion for the adaptively meshed model compared to the regular grid model. For our synthetic aquifer data set, the inversion using the adaptively meshed model completed in a quarter of the iterations used by the $0.5-\mathrm{m}$ square gridded model. In addition to a faster inverse result, the adaptively meshed model allows for more rapid testing of different inversion parameters and methodologies. Whereas our inversion uses a simple SIRT inversion algorithm, the inversion results may be improved by using a more sophisticated inversion algorithm or by including regularization. Translating existing regularization techniques or developing regularization techniques that can be applied to arbitrary meshes remains an area of research for adaptive-meshing inversion.

The adaptively meshed model creates an intuitive incorporation of the model's resolution into the final inversion product, and provides a visual representation of regions within the model that are better constrained by the data. If a conductivity change anomaly is located within an area of dense ray coverage, the adaptively meshed model will be able to better spatially resolve the shape of the anomaly because of the density of smaller cell sizes within high resolution areas. Adaptive meshing provides a method for altering the model resolution without adversely affecting the inversion method's ability to solve for conductivity change.

From our synthetic model, we predict that reflection-based GPR attenuation difference tomography can be used to detect conductivity changes on the order of tens of $\mu \mathrm{S} / \mathrm{cm}$. While the inversion algorithm could not match the magnitude of modeled conductivity changes, the inverted model does match the overall trend of conductivity change: regions with the greatest increase in conductivity were assigned larger conductivity change values than regions with less conductivity change. Reflection data is most suitable for imaging near-surface conductivity changes in the presence of multiple coherent reflectors. For many near-surface experiments, conductivity change estimates from reflection attenuation difference tomography should be comparable to crosshole tomography estimates.

\section{ACKNOWLEDGMENTS}

Funding for this work was provided by the U. S. Environmental Protection Agency (X-97008501-0), the USDA (NRI 2004-3510214802), and the Inland Northwest Research Alliance graduate fellowship program. Boise State University acknowledges support of 
this research by Landmark Graphics Corporation via the Landmark University Grant Program.

\section{REFERENCES}

Acworth, R. I., and G. R. Dasey, 2003, Mapping of the hyporheic zone around a tidal creek using a combination of borehole logging, borehole electrical tomography and cross-creek electrical imaging, New South Wales, Australia: Hydrogeology Journal, 11, 368-377.

Ajo-Franklin, J. B., J. A. Urban, and J. M. Harris, 2006, Using resolutionconstrained adaptive meshes for traveltime tomography: Journal of Seismic Exploration, 14, 371-392.

Aster, R. C., B. Borchers, and C. H. Thurber, 2005, Parameter estimation and inverse problems: Elsevier.

Barrash, W., and E. C. Reboulet, 2004, Significance of porosity for stratigraphy and textural composition in subsurface, coarse fluvial deposits: Boise Hydrogeophysical Research Site: GSA Bulletin, 116, 1059-1073.

Böhm, G., P. Galuppo, and A. Vesnaver, 2000, 3D adaptive tomography using Delaunay triangles and Voronoi polygons: Geophysical Prospecting, 48, 723-744.

Bradford, J. H., 2006, Applying reflection tomography in the postmigration domain to multifold ground-penetrating radar data: Geophysics, 71, no. 1 $\mathrm{K} 1-\mathrm{K} 8$.

K1 2007, Frequency-dependent attenuation analysis of ground-penetrating radar data: Geophysics, 72, no. 3, J7-J16.

- 2008, Measuring water content heterogeneity using multifold GPR with reflection tomography: Vadose Zone Journal, 7, 184-193.

Bradford, J. H., W. P. Clement, and W. Barrash, 2009, Estimating porosity with ground-penetrating radar reflection tomography: A controlled 3-D experiment at the Boise Hydrogeophysical Research Site: Water Resources Research, 45, 1-11.

Bradford, J. H., and J. C. Deeds, 2006, Ground-penetrating radar theory and application of thin-bed offset-dependent reflectivity: Geophysics, 71, no. 3, K47-K57.

Chambers, J. E., M. H. Loke, R. D. Ogilvy, and M. P. I., 2004, Noninvasive monitoring of DNAPL migration through a saturated porous medium using electrical impedance tomography: Journal of Contaminant Hydrology, 68, 1-22.

Chang, P., D. L. Alumbaugh, J. Brainard, and L. Hall, 2004, The application of ground penetrating radar attenuation tomography in a vadose zone infiltration experiment: Journal of Contaminant Hydrology, 71, 67-87.

Day-Lewis, F. D., J. W. Lane, J. M. Harris, and S. M. Gorelick, 2003, Timelapse imaging of saline-tracer transport in fractured rock using differenceattenuation radar tomography: Water Resources Research, 39, 12901303 .
Goodman, J. E., and J. O'ourke, 2004, Handbook of discrete and computational geometry: 2nd ed.: Chapman \& Hall/CRC.

Johnson, T. C., P. S. Routh, W. Barrash, and M. D. Knoll, 2007, A field comparison of Fresnel zone and ray-based GPR attenuation-difference tomography for time-lapse imaging of electrically anomalous tracer or contaminant plumes: Geophysics, 72, no. 2, G21-G29.

Kim, H. J., Y. Song, K. H. Lee, and M. J. Wilt, 2004, Efficient crosswell EM tomography using localized nonlinear approximation: Exploration Geophysics, 35, 51-55.

Kimmel, R., and J. A. Sethian, 1998, Computing geodesic paths on manifolds: Proceedings of the National Academy of Sciences, 95, 8431-8435.

Knight, R. J., and A. L. Endres, 2005, An introduction to rock physics principles for near-surface geophysics, in D. K. Butler, ed., Near-surface geophysics: SEG.

Kool M., N. Rawlinson, and M. Sambridge, 2006, A practical grid-based method for tracking multiple refraction and reflection phases in three-dimensional heterogeneous media: Geophysical Journal International, 167, $253-270$.

Lane, J. W., F. D. Day-Lewis, R. J. Versteeg, and C. C. Casey, 2004, Objectbased inversion of crosswell radar tomography data to monitor vegetable oil injection experiments: Journal of Environmental and Engineering Geophysics, 9, 63-77.

Larsen, R. M., 1998, Lanczos bidiagonalization with partial reorthogonalization: Ph.D. thesis, Aarhus University.

Levander, A. R., 1989, Finite-difference forward modeling in seismology, in D. E. James, ed., Encyclopedia of Solid Earth Geophysics: Van Nostrand Rheinhold, 410-431.

Liu, L., J. W. Lane, and Y. Quan, 1998, Radar attenuation tomography using the centroid frequency downshift method: Journal of Applied Geophysics, 40, 105-116.

Rawlinson, N., and M. Sambridge, 2004, Multiple reflection and transmission phases in complex layered media using a multistage fast marching method: Geophysics, 69, 1338-1350.

Rüger, A., and D. Hale, 2006, Meshing for velocity modeling and ray tracing in complex velocity fields: Geophysics, 71, no. 1, U1-U11.

Sethian, J. A., and A. Vladimirsky, 2000, Fast methods for the eikonal and related Hamilton-Jacobi equations on unstructured meshes: PNAS, 97, 5699-5703

Stork, C., 1992, Reflection tomography in the postmigrated domain: Geophysics, 57, 680-692.

Taner, M. T., F. Koehler, and R. E. Sheriff, 1979, Complex seismic trace analysis: Geophysics, 44, 1041-1063.

Wharton, R. P., G. A. Hazen, R. N. Rau, and D. L. Best, 1980, Electromagnetic propagation logging: Advances in technique and interpretation: 55th Annual Fall Technical Conference and Exhibition, SPE.

Zhang, H., and C. H. Thurber, 2007, Estimating the model resolution matrix for large seismic tomography problems based on Lanczos bidiagonalization with partial reorthogonalization: Geophysical Journal International, 170, 337-345. 